\title{
INTERACTIVE VISUALIZATION OF COMPLEX THREE-DIMENSIONAL MODELS: CASE STUDY IN ZEBRAFISH
}

\author{
Feibo Duan, Kristian F. D. Rietveld and Fons J. Verbeek \\ Leiden Institute of Advanced Computer Science (LIACS), Leiden University, \\ Niels Bohrweg 1, 2333 CA Leiden, The Netherlands
}

\begin{abstract}
Modern microscope imaging techniques can generate three-dimensional (3D) images in high numbers. Understanding of the content of such images requires visualization and some form of interaction with the content. In this paper a generic processing pipeline from two-dimensional (2D) microscopy data to interactive visualization of complex 3D models is presented. This pipeline supports 3D images reconstructed from different microscopy modalities and interactive visualization using various display devices and interaction methods. Using an annotated zebrafish model containing 188 substructures as a case study, we illustrate the importance of developing suitable visualization and interaction methods to empower researchers better understand and analyze the problem at hand.
\end{abstract}

\section{KEYWORDS}

Interactive Visualization, 3D Models, 3D Visualization, Bioimages

\section{INTRODUCTION}

Modern microscope imaging techniques are capable of rapidly acquiring and generating three-dimensional (3D) imagery and it is expected that with current developments there will be a further decrease in acquisition time. Data acquisition methods in optical microscopy that are used to this end are confocal laser scanning microscopy (CLSM), optical projection tomography (OPT) and serial sectioning. In all above three cases two-dimensional (2D) images are acquired from which a 3D volumetric image is reconstructed.

Zebrafish model system is widely used in biomedical research. George Streisinger at the University of Oregon first used zebrafish as a model system in the late 1960s, biologists thereafter are increasingly using zebrafish in their research (Grunwald \& Eisen, 2002). Zebrafish offer many advantages in biomedical research such as high fecundity, small size, short generation time, external fertilization, and numerous transparent specimens.

Sophisticated 3D visualization of zebrafish is in high demand. This has led to more general availability of 3D images. During the data analysis process, researchers have a need to get more detailed information such as organ detail, a system detail, entire object, spatial relationship of different parts of whole object and so on. To support this, substructures in 3D images can be segmented and annotated by hand or in an automatic fashion (Tang, 2020). These developments call for improved visualization and interaction methods to help researchers better understand and analyze the problem at hand. To this end, a visualization system is required that is flexible in the selection of parts that are visualized, in contrast to conventional visualization methods that only show the whole model or single specific parts. To facilitate the study of these more complex and more detailed 3D models, multiple interaction methods should be provided, as a regular desktop monitor and mouse used by conventional systems are often not well suited.

To solve these problems, we propose a generic processing pipeline from $2 \mathrm{D}$ raw data to interactive visualization of complex 3D models, which supports 3D models generated using different microscopy modalities and allows interactive visualization of 3D models on various display systems. 


\section{OVERVIEW OF THE PIPELINE}

Our focus is on the visualization of 3D models that are obtained from 3D reconstructions of 2D microscopy data. Our intention is supporting 3D models acquired through different modalities and visualization with various interaction methods. To achieve this, we propose a generic processing pipeline from 2D raw data to visualization of a complex 3D model. This pipeline, depicted in Figure 1, consists of three main components: 3D volumetric image acquisition, surface model generation ("mesh"), and interactive visualization.

In this paper, we use zebrafish model system as a case study. With our OPT system, 400 2D images acquired from the specimen are reconstructed to a 3D volumetric image through cropping, background subtraction, center of rotation correction and 3D reconstruction (Tang et al., 2017). Note that 3D data generated using other modalities will also work in our processing pipeline, as indicated in Figure 1. Subsequently, an optional segmentation step generates and labels all substructures of a model, to support visualization of and interaction with substructures. As the next step a surface model is generated from the 3D volumetric image using contour extraction and surface reconstruction which uses the Boissonnat method (Boissonnat \& Geiger, 1993). Finally, this surface model is visualized by an interactive visualization application that works on different display devices employing different interaction methods.

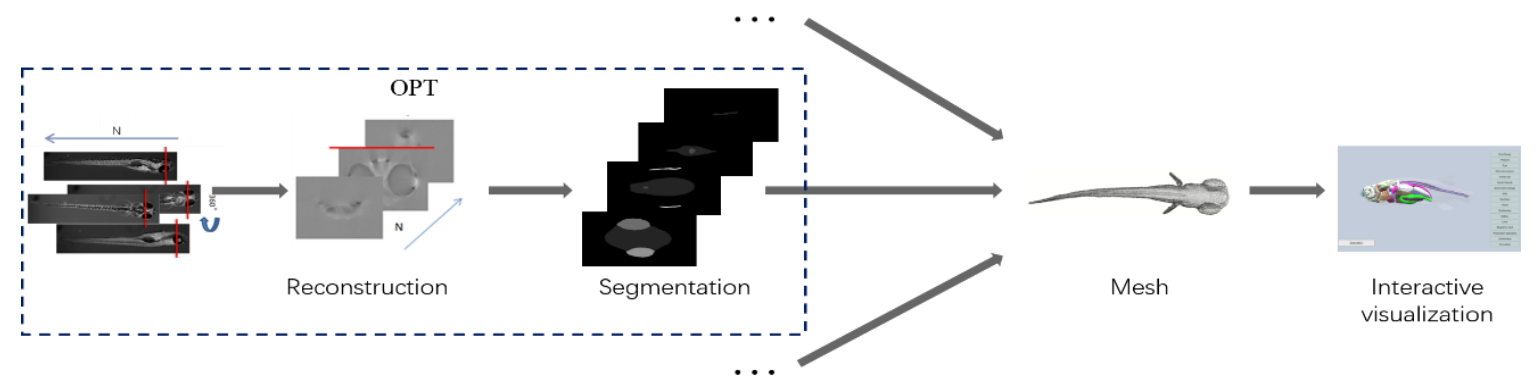

Figure 1. The generic processing pipeline from 2D raw data to interactive visualization of a complex 3D model

\section{GENERATION OF SURFACE MODELS}

The 3D models that are the result of the reconstruction processing pipeline are volume models. Such models are not very suited for rapid 3D rendering, in which case surface models are typically used. Therefore, our processing pipeline applies contour extraction followed by the Boissonnat method to generate surface models. However, this might lead to the situation where the surface of the 3D object is incomplete, as shown in Figure 2(a). This is caused by the fact that some face normals are inverted. When the direction in which light is emitted is the same as the direction of the face normal, then, in the computational sense, light does not reflect in the user's eye. To alleviate this problem there are two possibilities: either all face normals are adjusted to the correct direction, or a rendering method is chosen that renders both sides of the surface. To consider inclusiveness of different surface models, double-sided rendering is used in our case. The result is shown in Figure 2(b), which is clearly an improvement.

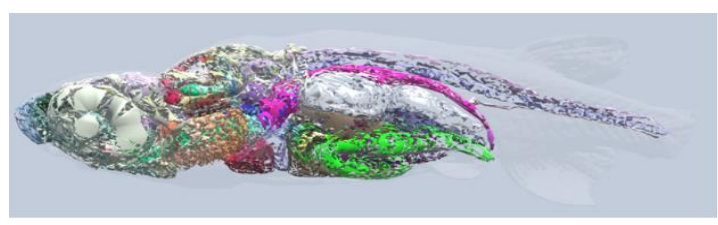

(a)

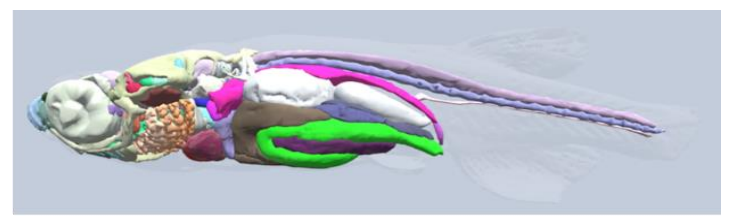

(b)

Figure 2. Display results: (a) standard rendering and (b) double-sided rendering 


\section{INTERACTION VISUALIZATION ON DIFFERENT DEVICES}

To design and implement an interactive visualization application of complex 3D models that can run on different display devices and support different interaction methods, Unity 3D is used as a development platform. The application that we have developed is generic in nature and has two main features: 1) It can show substructures, i.e. organ systems with respect to the entire structure, so that a user can observe position relationships of several organs or parts without occlusion. 2) It allows a user to select a part of the object and interact with it. To enable manipulation of substructures, separate surface reconstructions of annotated parts of a complex 3D model are loaded into the Unity project.

As can be seen in Figure 3, the interactive visualization application that we have developed supports various display devices: i.e. regular computer monitor, HD video wall, Virtual Reality (VR) system, hologram system and smartphone. The available interaction methods differ per display device; a mouse is used with a regular computer monitor, VR systems use specific input methods and smartphones employ touch screens. Some display devices, such as a video wall, support multiple input methods. Whereas a mouse is used with a regular computer monitor, a mouse is not suitable for interaction with a model displayed on a video wall. To improve interaction with the video wall, we are experimenting with the WiiMote, Leap Motion and Kinect controllers. The generic implementation of the visualization pipeline described in this paper allows us to evaluate all of these different interaction methods with ease.

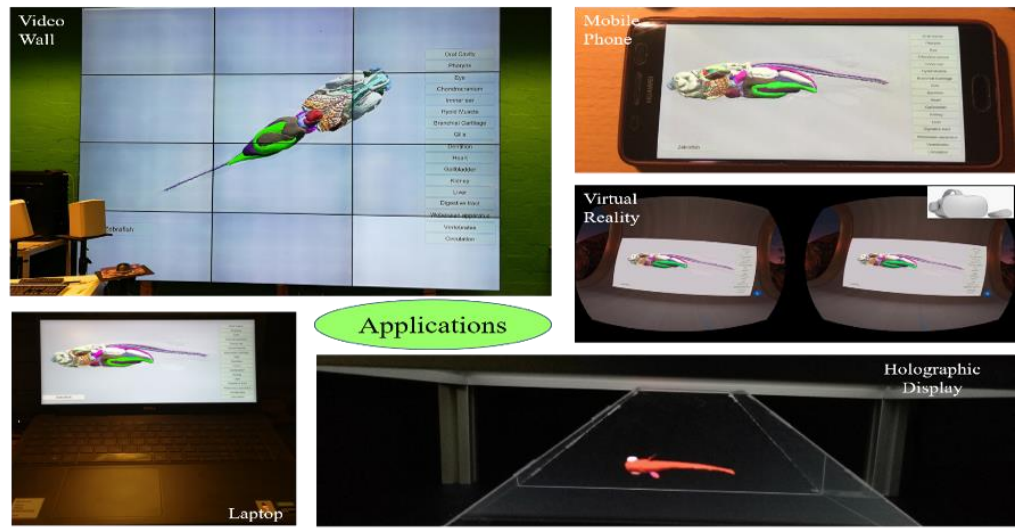

Figure 3. Interactive visualization on different platforms

\section{PRELIMINARY RESULTS}

To illustrate the generic processing pipeline that has been developed, we present the interactive visualization of 3D zebrafish as a case study. Our complex 3D model of juvenile zebrafish contains 188 annotated structures that are displayed separately in the interface. The large amount of structures is organized in an ontology so that they can also be manipulated as groups. This makes the visualization very versatile. Figure 4(a) shows an entire zebrafish model with substructure options (the zebrafish skin is rendered transparent). In Figure 4(b), the eyes substructure has been selected. Users can observe the spatial information of eye parts whereas the other parts of the zebrafish are now rendered semi-transparent. As is shown in Figure 4(c), it is also possible to select one particular substructure and hide the other parts of the model. Finally, Figure 4(d) shows that users can also obtain an exploded view that provides all substructures and their annotation. In all of these different modes, the user can interact with the displayed objects through rotation, zooming and translation using a controller suitable for the display device at hand. 


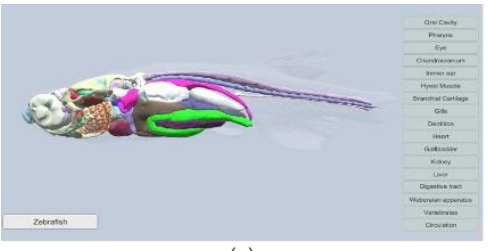

(a)

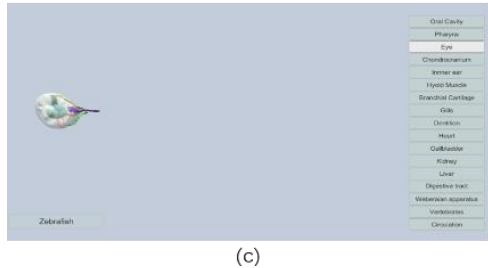

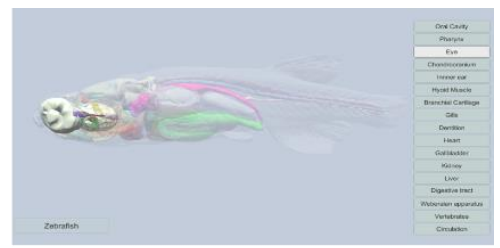

(b)

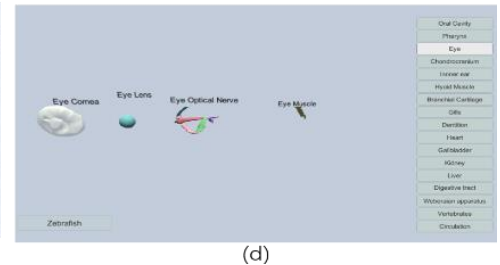

(d)

Figure 4. Interactive visualization of a 3D zebrafish model using different display modes

\section{CONCLUSIONS}

In this paper we have described a generic processing pipeline starting from coherent 2D microscopy raw data to interactive visualizations of complex 3D models. The intention of the processing pipeline is to separate the device dependent from the generic. To that end, we distinguish imaging device dependences, display device dependences and interaction device dependences. The preparation of the 3D model is considered generic. Subsequently, specific applications can be generated for each visualization platform. Using an annotated zebrafish model as a case study, we have developed an application that runs on different platforms employing different interaction methods. In our future work, we will further experiment with different interaction devices as well as supporting 3D reconstructions that have been created with other imaging modalities. Moreover, the surface reconstruction method will be further improved to enhance image quality, for instance by getting an optimized surface mesh from a point cloud (Cao \& Verbeek, 2013) which is derived from the initial surface mesh.

\section{ACKNOWLEDGEMENT}

This research was partially supported by the China Scholarship Council (CSC). We thank Robin Megens for his work on the annotation of a juvenile Zebrafish.

\section{REFERENCES}

Boissonnat J., Geiger B., 1993. Three dimensional reconstruction of complex shapes based on the Delaunay triangulation. Proc. SPIE 1905, Biomedical Image Processing and Biomedical Visualization, San Jose, CA, United States, pp. 964-975.

Cao L., Verbeek F.J., 2013. Analytical evaluation of algorithms for point cloud surface reconstruction using shape features, J. Electron. Imaging, Vol. 22, Issue. 4, 043008.

Grunwald D. J., Eisen J. S., 2002. Headwaters of the zebrafish -- emergence of a new model vertebrate, Nat Rev Genet, Vol. 3, Issue. 9, pp. 717- 724.

Tang X. et al, 2017. Fast Post-Processing Pipeline for Optical Projection Tomography, IEEE transactions on nanobioscience, Vol. 16, Issue. 5, pp. 367-374.

Tang X., 2020. Computational optimisation of optical projection tomography for 3D image analysis, PhD thesis, Leiden University, Leiden. 